\title{
The Role of Immunity in Fighting and Checking the Spread of Viral Diseases
}

\begin{abstract}
Raghavendra Rao MV ${ }^{1 *}$, Srinivasa Rao D², DilipMathai ${ }^{3}$, Jithendra Kumar Naik ${ }^{4}$, Adarsh Meher Nisanth ${ }^{5}$, Mahendra Kumar Verma ${ }^{6}$, Vijay Kumar Chennamchetty ${ }^{7}$ Ahmad Abdul Khabeer ${ }^{8}$ and Khizer Hussain Junaidy ${ }^{9}$
\end{abstract}

${ }^{1}$ Director of Central Research laboratory, Apollo institute of Medical Sciences and Research, Hyderabad, TS, India

${ }^{2}$ Department of Biotechnology, Acharya Nagarjuna University, Nagarjuna nagar, Guntur, Andhra Pradesh, India

${ }^{3}$ Department of Medicine, Dean, Apollo Institute of Medical Sciences and Research, Jubilee Hills, Hyderabad, Telangana, India

${ }^{4}$ Departmnet of Zoology, Principal, University college of Science, Osmania University, Hyderabad, India

${ }^{5}$ NRI Medical College, Dr.NTR University Health Sciences, Vijayawada, Andhra Pradesh, India.

${ }^{6}$ American University School of Medicine Aruba, Caribbean islands

${ }^{7}$ Department of Pulmonary Medicine, Apollo Institute of Medical Science and Research, Hyderabad, TS, India

${ }^{8}$ ENT, Gandhi Medical college, Hyderabad, TS, India

${ }^{9}$ Department of Pharmacology, Gandhi Medical College, Hyderabad, TS, India

*Corresponding author: MV Raghavendra Rao, Scientist-Emeritus and Director Central Research laboratory, Apollo institute of Medical Sciences and Research, Hyderabad, TS, India.
Received Date: January 19, 2021

Published Date: February 08, 2021

\section{Abstract}

Immunology is truly a fascinating discipline that meets the challenge and offers opportunity in fighting and checking the spread of diseases. Many viruses infect humans. The host response to invading virus depends upon the infectious agents and where it is encountered. In response to virus entry there may not be, always, an overt reaction leading to clinical manifestation. There may be simply, subclinical infection, which would protect the individual from later exposure. A number of specific immune effector mechanisms together with non-specific defence mechanism play role in eliminating an infective virus.

Viruses are intracellular parasites. Many viruses have developed very effective escape mechanisms. Influenza virus which has acquired various types of antigens associated with their capsids. The outer envelope of influenza virus has numerous spikes, which are linked to pathogenicity and antigenicity of the strain. Gene recombinations cause antigenic shifts, producing new strains. Certain viruses like rubella causing measles produce immunosuppressive chemicals. Varicella zoster and a number of herpes virus groups, which replicate in the upper respiratory tract, remain latent in the sensory ganglia of spinal and cranial nerves. In these places the viruses do not come in contact with antibodies, hence remain protected. EpsteinBarr virus has Complement receptor 2 (CR2), Influenza virus has Sialic residue on cell surface glycoprotein, Rhinovirus contains Intracellular adhesion molecule( ICAMs ) and Vaccinia virus with Epidermal growth factor receptor C for virus entry. The fact that antibody protects against some virus infection is attested by the widespread use of immunoglobulin for prophylaxis against measles, and by the world wide efforts made to stimulate antibody production by immunization against poliomyelitis viruses.

Keywords: Intraepithelial neoplasia(CIN); Tumor antigen-specific cytotoxic T lymphocyte (CTL); G-CSF (Granulocyte colony-stimulating factor); Human papillomavirus (hrHPV); Cytomegalovirus (CMV); Complement receptor 2 (CR2); Intracellular adhesion molecule( ICAMs ) 


\section{Introduction}

Responses to infected cells by different types of CD4+ T cells orchestrate a tissue-damaging inflammatory reaction and these become chronic against persistent viruses. Most often, the cell subsets involved are T helper 1 (TH1) cells, but TH17 cells may contribute to inflammatory responses during HIV, HCV and influenza virus infections [1-4].

TH2 cells are rarely associated with inflammatory responses during viral infections [5]. Adenovirus can cause heart muscle damage, left ventricular dysfunction, heart muscle dysfunction, and severe heart transplants. Coxsackie B virus induces cardiomyopathy and damages heart cells. The immune system continues to damage the heart. This virus extensively damages the heart. Cardiovascular complications constitute a rare manifestation of Cytomegalovirus (CMV) infection; it is a diagnostic challenge in emergency cases [6].

There are 4 types of dengue viruses.(DEN 1,2,3, and 4) and the presence of antibody directed against one type does not give cross-protection against the other three, worse still is the fact that antibodies can enhance the infection of peripheral blood mononuclear cells by Fc-receptors, mediated uptake of the antibody-coated dengue virus particle [7]. Standard serological differentiation of acute (DENV) infection using immunoglobulin $\mathrm{M}(\operatorname{IgM})$ and $\operatorname{IgG}(\operatorname{IgG})$ assays demonstrated high false positive rates, particularly in vaccinated individuals [8].

Serological assays detecting ant dengue immunoglobulin M (IgM) or immunoglobulin G (IgG) antibodies, with or without dengue non structural protein 1 (NSI) antigen detection are widely used for dengue diagnosis,seroprevalence analysis and disease surveillance as such tests are user friendly and affordable.However the utility of these serological assays may be confounded by dengue vaccination as IgG and IgM levels may reflect vaccine -induced responses [9]. Chemokine-like IL-8 (interleukin-8) and colony-stimulating factors like G-CSF (Granulocyte colony-stimulating factor) promote chemotaxis and neutrophils maturation respectively [10,11]. IL-6 is a potent pyrogenic cytokine, trafficking to lymphoid organs during febrile events [12] IL-6 has been implicated in the progression of several virus infections [13] IL-6 during viral infections has been gathered in experimental infections using IL-6-deficient mice $[14,15]$ IL-1 stimulates B-cell proliferation, differentiation and synthesis of immunoglobulins [16].

Mechanisms of fever induced by recombinant human interferon Certain symptoms of infections, such as fever, muscle pain and "flulike symptoms", are also caused by the production of IFNs and other cytokines [17] T cell activation is important in hrHPV infection. It has been proven that in patients [18] The induction of inefficient CD4+ T cells by high risk human papilloma virus (hrHPV) infection promots Cervical intra epithelial neoplasia (CIN) lesion progression [19]. Distorted equilibrium between type 1 T-helper cells (Th1) and Th2 cells is another property of cellular immunity during hrHPV infection [20]. Increased Th2 cytokine (IL-10) and reduced Th1 cytokine (IFN- $\gamma$, IL-12, IL-2 and tumor necrosis factor- $\alpha$ ) levels have been detected in cervical exudates of hrHPV+ patients [21,22].

\section{Chronological Record of Significant Events}

Based on practical understanding that immunity is produced after the attack of a disease

- Edward Jenner--------------------- Immunity against smallpox.

- Louis Pasteur---------------------- Coined the term vaccine

- Metchnikoff------------------- Importance of cells in immunity

- Paul Ehrich------------------------- Side chain hypothesis

- $\quad$ Emil Von Behring------------------Humoral theory

- Bordet. ----:---------------- Alexin as complement

- $\quad$ Robert Koch-------------------- Cellular immunity

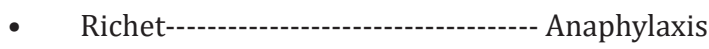

- $\quad$ Land steiner----------------------- Blood groups

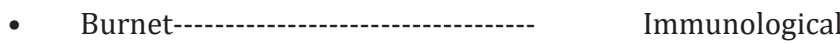
tolerance

- $\quad$ Edelman and Porter--------------------Nature and structure of Antibody

- Yallow--- -

- $\quad$ Benacerraf, Snell and Dauset----------MHC genes

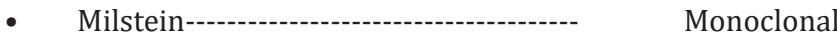
antibody

- Morell, B Roth-Wicky, F Skvaril ---Human Immunoglobulin-G

\section{Innate Immune Response to Viral Infection}

Early non-specific or innate immune - Interferon (IFN), Type I IFNs (IFN $\alpha$ and IFN $\beta$ ), Type II IFN (IFN $\gamma$ ), Natural killer cells and Macrophages

\section{Interferons}

Interferon (INF) is used for several unrelated class of proteins, which have antiviral effects some of them are glycosylated. Interferons may be produced by viral infected cells or a sensitised lymphocyte encountering the cell.Interferon alters cellular metabolism so that further assembly of viral particle is prevented. Interferons (IFNs) are a family of cytokines that were first identified almost half a century ago through their antiviral properties [23].

A family of low molecular weight proteins produced by eukaryotic cells having antiviral effects has attracted considerable 
attention in recent years. These are collectively known as interferons. Interferons are a unique class of antiviral agents which are discovered by Isaacs and Lindermann in 1957.They are produced by microbial pathogens also which reproduce within the host cells. Interferons also produced in minute quantities by infected cells as well as certain lymphocytes. After they are released,t hese substances migrate to uninfected cells in order to give protection against viral infection [24]

\section{Type-1 Interferons}

\section{Interferon-Alfa ("IFN- $\alpha$ ")}

IFN- $\alpha$ has been used for treatment of hepatitis C, hepatitis $B$, and in several cancers, including hairy cell leukemia, chronic myelogenous leukemia, and Kaposi's sarcoma [24]. IFN- $\alpha$ is produced by Plasmacytoid dendritic cells. natural interferon alpha (IFN- $\alpha$ ) obtained from the leukocyte fraction of human blood following induction with Sendai virus. Interferon alfa contains several naturally occurring IFN- $\alpha$ subtypes and is purified by affinity chromatography. The major subtypes identified are IFN- $\alpha 1$, IFN- $\alpha 2$, IFN- $\alpha 8$, IFN- $\alpha 10$, IFN- $\alpha 14$ and IFN- $\alpha 21$. Of these, IFN- $\alpha 2$ and IFN- $\alpha 14$ are glycosylated. The IFN- $\alpha$ content is expressed in International Units per milliliter, and the drug product is formulated in isotonic phosphate buffer solution at $\mathrm{pH}=7.2$, and supplemented with human albumin at $1.5 \mathrm{mg} / \mathrm{ml}$

\section{Interferon-Beta (IFN- $\boldsymbol{\beta}$,)}

- $\quad$ IFN- $\beta$, perhaps through its inhibition of IL-12 production as well as induction of IL-10, an immunosuppressive cytokine, is the first drug shown to promote clinical improvement in MS.

- $\quad$ IFN $-\gamma$ Interferon type II (IFN- $\gamma$ in humans): This is also known as immune interferon and is activated by Interleukin-12 [26]. IFN type II binds to IFNGR, which consists of IFNGR1 and IFNGR2 chains [27].

\section{Phagocytic Cells of the Innate Immune Response}

\section{Macrophages}

Macrophages are the large eaters. These are the long lived phagocytes. These cells move over alveolar surfaces, scavenger dust particles, microorganisms and other debris. The attachment of antigens to macrophage is specific. All the macrophages have specific receptors for $\mathrm{C} 3$ component of complement as well as Fc component of antibody

The C3 receptor promotes the adherence of antigen to macrophage by way of opsonization antigen by the complement whereas the Fc receptors help in binding within the antibody there by promoting the phagocytosis of antigen antibody complex. When the antigen adheres with lymphocyte processing, receptors for the antigen, recognition takes place and thus the lymphocytes are induced to produce immunity.

The macrophages that present antigens to T-helper cells (Th) should have MHC determinant of class 11 on the surface whereas macrophage that present antigens to T-cytotoxic cells (Tc) cells should have the MHC determinant, it cannot cooperate and thus antigen presentation cannot occur, which is known as MHC restriction Macrophages are important secretory cells producing and secreting a number of substances such as components of complement system, hydrolytic enzymes, toxic forms of oxygen and the monokines. Themonokines have regulatory effects on lymphocyte function. Each cell type expresses characteristic surface molecules (CD3,CD4,CD8.) These cells scavenging the dust particles, microorganisms and other debris. The primary function of macrophage is phagocytosis. The macrophages, by their property of amoeboid movement, put forth pseudopodia which help in engulfing any solid particle such as the invading microorganisms. The attachment of antigens to macrophage is specific. All the macrophages have surface receptors for C3 component of complement as well as for Fc component of antibody. Phagocytosis of virus and virus-infected cells; Killing of virus-infected cells; and Production of antiviral molecules: TNF $\alpha$, nitric oxide, and IFN $\alpha$.

\section{Neutrophils}

Neutrophils only migrate into tissue if there is inflammation. Monocytes cruise normal tissue When macrophages encounter a signal (infection), they recruit neutrophils to the site!. Example, mediate selectins and adhesins which cause neutrophils to stop and migrate into tissue The term 'left shift' indicates that the neutrophils present in the blood are at a slightly earlier stage of maturation than usual. Inflammatory signaling Mediators produced by macrophages and neutrophils are Prostaglandins.

Lipid derivatives of arachidonic acid they Inhibit platelet aggregation, increase vascular permeability, induce smooth muscle contraction Leukotrienes Lipid derivative of arachidonic acid slow reactive substance of anaphylaxis - SRS-A -But longterm inflammation (chronic) is controlled by macrophages -If not cleared, chronic inflammation turns into a granuloma

\section{Mast cells and Basophils Cells}

These concentrated within the respiratory and gastrointestinal tract, and within the deep layers of skin. Influenced by TH2, and IL-13 and IL-4 Reside in submucosa, skin, connective tissue Numbers in tissue increase during worm infection IgE binds to Fc receptors on the surface of mast cells Binds to IgE Even though Ig is not bound to antigen Receptor is FceRI One mast cell has multiple specificities Cross-linking of IgE molecules à activation (Mast cells also activated by $\mathrm{C} 3 \mathrm{a}, \mathrm{C} 5$ and certain drugs, STAY TUNED) Activation à degranulation and synthesis of mediators Mast cell proteolytic enzymes = tryptase and chymotrypsin These enzymes Increase mucus production -Increase smooth muscle contraction. Cleave and activate complement components and kinin components à inflammation Histamine (à pruritus) .Smooth muscle contraction -Increased vascular permeability .Chemotactic for white cells Cytokines to promote and extend inflammation .TNF àlfa enhanced diapedesis à inflammation -IL-4 stimulates 
TH2 responses -IL-3 and IL-5 stimulates eosinophil production and activation Eosinophils These cells contain Blobbed Nucleus These are granulocytes. Phagocytic. Granules contain hydrolytic enzymes. Primary defense against helminth infections (MBP) Eosinophils contains Toxic substances in granules. Derived from the same precursor as PMNs. Influenced by IL-3 and IL-5 (secreted by TH2 cells) Leukotriene are produced by mast cells. Provokes bronchospasm Cationic protein à damages worm plus damages worms nervous system (a neurotoxin) Neutralizing antibodies

\section{IgA}

IgA found only to a small extent in serum. IgA predominantly seen in the external seromucous secretions of the respiratory, gastrointestinal and urogenital tracts. IgA is the second abundance among the immunoglobulins. IgA has two sub classes. Iga1 and IgA2.IgA present in the colostrum protects the baby from intestinal pathogens. It will not fix complement but is found to activate the alternative complement pathway there by neutralizing local toxins. IgA is the complement of the secondary humoral response. IgA cannot cross placenta.

\section{IgG}

IgG activates the classical component pathway during antigen antibody reactions. IgG neutralizes toxins and viruses Immunoglobulin M (IgM) is one of several iso types of antibodies (also known as immunoglobulin) that are produced by vertebrates $[28,29]$. In the case of humans and other mammals, the spleen is is the major site of specific IgM production [30,31].

\section{Immune complex reactions}

Immune complex reactions are induced by deposition of antigen and antibody in the tissues, causing the activation of complement, this results in a polymorphonuclear inflammatory response and also damage to the cell membranes of adjacent tissues. In addition to the effect of complement, macrophages and lymphocytes are activated by the attachment to the Fc component o0f immunoglobulin molecule. Hydrolytic enzymes released from granules of the leukocytes also contribute to the vascular damage which is the hallmark of immune complex reaction.

\section{ADCC}

Antibody-dependent cellular cytotoxicity (ADCC), also referred to as antibody-dependent cell-mediated cytotoxicity, is a mechanism of cell-mediated immune defense whereby an effector cell of the immune system actively lyses a target cell, whose membranesurface antigens have been bound by specific antibodies [32]. Antibodies, as part of the humoral immune response, can act to limit infection [33]. ADCC requires an effector cell which classically is known to be natural killer (NK) cells that typically interact with immunoglobulin G (IgG) antibodies [34]. However, macrophages, neutrophils and eosinophils can also mediate ADCC, such as eosinophils killing certain parasitic worms known as helminths via IgE antibodies [35].

\section{Cytotoxic T-cells and MHC restriction}

There is evidence for Tc cells in some virus infections. Vaccination of man with killed whole influenza A results in an increased ability to mount an in vitro HLA restricted Tc cell response against cells infected with the same or different strains. The lack of strain specificity agrees with several reports that in mice Tc cell generated by one variant can protect against serologically distinct variants. This may prove important influenza A virus avoids the antibody response by changing its surface haemagglutinin and neuraminidase glycoproteins every few years Antibodies to these glycoproteins are type specific and so do not block infection with antigenic variants.Note that HLA restriction implies that Tc cells are involved un like the K cell activity.

Cytotoxic T lymphocytes (CTLs) and Helper $\mathrm{T}$ (Th) cells help in adaptive immune response to viral infection. The Tc cells recognize the antigenic peptides(virus infected cells, tumor cells) in association withMHC-1 molecules. The peptide -MHC-1 complex is the first signal for the activation of Tc cells..The second signal is offered by IL-2secreted by near Th cells. Following by these two signals, activation of Tc cells lead to the destruction of virus infected cells, tumor cells or foreign cells(in the form of transplant [36].

Cytotoxic T cells (CTL) kill infected cells. They are identified by the surface marker CD8-(CD8+ T-cells). Also require cell to cell contact to bind antigen. Bind only processed antigen. Secrete cytokines and chemokines.

\section{Delayed hypersensitivity to viral antigens}

The difference between CD8+ T cells and CD4+ T cells mediating DTH relates to the molecular mechanisms by which antigens are processed and presented to the T cells [36].

\section{Protection by antibody in virus infections}

The fact that antibody protects against some virus infections is attested to by the widespread use of immunoglobulin for prophylaxis against measles, and by worldwide efforts made to stimulate antibody production by immunization against poliomyelitis viruses. Nevertheless the role of antibody in protection against some other virus infections is far from obvious. In order to understand these differences it is necessary to consider some general points about virus infections. these have only become obvious in recent years, as a result of the greatly improved techniques for isolating virus from tissue even when present in small amounts. The first important point to recognize is that infection can occur without recognizable clinical symptoms. Protection by antibody is usually considered in terms of protection against overt disease. The second important point is that virus infections can, by and large, be divided into two groups.

A. Those in which the portal of entry, and the target organs, are at the surface, eg, influenza and common cold

B. Those virus infections in which the portal entry is probably the oropharynx, but in which clinical symptoms do not occur 
until dissemination through the bloodstream has taken place and in which the target organs are different from portal entry, eg poliomyelitis, measles ,hepatitis, chickenpox and smallpox [38].

\section{Mechanism of Immune Evasion by Viruses}

1. Antigenic variation

---------- -Influenza, rhinovirus, HIV

2. Inhibition of antigen processing Blockade of TAP transporter and

Removal of class I molecules from the ER
(HSV)

Cytomegalovirus (CMV)

3. Production of "decoy" MHC molecules to inhibit NK cells--- -------Cytomegalovirus (murine)

4. Production of cytokine receptor
homologues--------------------- Vaccinia, poxviruses (IL-1,

IFN- $\gamma$ ) Cytomegalovirus (chemokine) (39)

\section{Immunity in Rotavirus}

Vomiting is a hallmark of rotavirus disease. Local immune factors such as secretary IgA or interferon, may be important in protection against rotavirus infection. Alternately, reinfection in the presence of circulating antibody could reflect the presence of circulating antibody could reflect the presence of multiple serotypes of virus Rotavirus antibody has been detected in colostrum for up to nine months postpartum.

The mechanism responsible for generating protective immunity to rotavirus infections and illness following vaccination or natural infection are not completely understood, especially in humans where detailed examination of the acquired cellular immune response ( $\mathrm{B}$ and $\mathrm{T}$ cells) in young children has been limited because of the difficulty in obtaining timely and sufficient specimens. The contribution in innate immunity to control the rotavirus infection has only recently been examined, and these studies have been primarily in animal models [40].

\section{Immunity in Cytomegalovirus}

Antibodies occur in most human sera virus may occur in urine of children for many months even though serum Nt antibody is present. This suggests that the virus propagates in the urinary tract rather than being filtered from the bloodstream. Virus is not found in young children who lack antibody

\section{Immunity in Human Papillomavirus}

Cervical cancer is the second most common cause of cancer worldwide and the primary cause of cancer in women in developing countries [41]. A study in the united states look at the uptake of HPV vaccine show only 1 out of 3 girls (11-17 Years) have received greater than or equal to one dose of HPV vaccine and much less have completed all the doses they suggested that patient education about the vaccination is necessary to increase vaccine uptake among girls, especially those 11-12 Years olds [41].

\section{Immunity in EB (Epstein-Barr virus)}

The most widely used and most sensitive serological procedure for detection of EB virus infection indirect immunofluorescent test with acetone-fixed smears of cultured Burkitt's lymphoma cells The cells containing EB virus exhibit fluorescence after treatment with fluorescent antibody. Detectable levels of antibody persists for many years.

\section{Immunity in Varicella,HSV-1 and HSV-2}

Varicella and Zoster viruses are identical, the diseases being the result of differing host responses. Previous infection of varicella leaves the patient with enduring immunity to varicella. However zoster may occur in persons who have contracted varicella earlier. This reactivation of a varicella virus infection has been latent for years. Many newborns positively transferred maternal antibodies. The antibody is lost during the first six months of life, and the period of greatest susceptibility to primary herpes infection occurs between ages 6 months to 2 years.Type- 1 antibodies begin to appear in population in early chidhood, by adolescence, they are present in most persons..Antibodies to type-2 rise during the age of adolescence and sexual activity [43].

\section{Immunity in Hepatitis-C}

Hepatitis $C$ virus belongs to the flavivirus family. Hepatitis $C$ virus is known as Non-A and Non-B hepatitis.

It can also be considered as STD and can be transmitted at birth from an HCV infected mother. Acute disease is usually mild but $70 \%$ of those infected develop chronic disease. Treatment with ribavirin and pegylated polyethylene Glycerol recombination therapy can rid the virus in $50 \%$ of those infected with genotype 1 and in the $80 \%$ of those infected with genotype 2or3 [44] The advanced discovery of direct-acting antivirals (DAAs) is highly useful in the treatment of chronic hepatitis $\mathrm{C}$ infection. Direct acting antivirals are now useful in the elimination of HCV scrupulously. The advanced discovery of direct-acting antivirals (DAAs) is highly useful in the treatment of chronic hepatitis $\mathrm{C}$ infection

\section{Immunity in Hepatitis--B}

Jaundice is a disease that your friends diagnose. Hepatitis B can cause mild illness lasting a few weeks, or it can lead to a serious, lifelong illness. The virus infects hepatic cells and causes liver tissue degeneration and release of liver associated enzymes (transaminases) into blood stream. This is followed by jaundice, accumulation of bilirubin (A black down product of hemoglobin) in skin and other tissues with resulting yellow appearance. The first Hepatitis-B- vaccine for active immunization, introduced in 1982 , was prepared from purified non-infectious $22 \mathrm{~nm}$ spherical HBsAg particles derived from plasma of healthy carriers. In 1987, the plasma derived vaccine was a genetically engineered vaccine 
derived from recombinant yeast. The plasma-derived vaccine consists of purified inactivated HBsAg particles obtained from the plasma of chronic carriers. Now only the DNA recombinant vaccine is available. In this, the antigen particles are obtained from the yeast Saccharomyces cerevisiae through recombinant DNA technology.

\section{Immunity in Human immunodeficiency virus}

Acquired immune deficiency syndrome (AIDS) is a disease caused by immune deficiency virus. HIV spread through certain body fluids that attack the body's immune system, specifically CD4 cells, often called T cells. Over time HIV can destroy so many of these cells that the body can't fight off infections and disease. These special cells help the immune system fight off infections. Untreated, HIV reduces the number of CD4 cells (T cells) in the body.

- $\quad$ CD4 less than 500-TB,Herpes zoster, Candida albicans

- $\quad$ CD4 less than 200-Pnemocystis Jiroversi pneumonia

- $\quad$ CD4 less than 100---Toxoplasmosis, Cryptococcosis, CMV infection

- Cancer-Kaposis sarcoma, Non Hodgkin's lymphoma Cervical carcinoma [45].

\section{Immunity in Influenza}

Influenza is a specific acute illness caused by a group of myxoviruses. In this, immunity is a type specific and if the antigenic constitution of a new strain can be detected early,a specific vaccine may give about $70 \%$ protection. Annual winter vaccination is recommended for patients suffering from chronic pulmonary, cardiac or renal disease [46].

After an attack of influenza, the ensuing immunity persists for an year or more and confers resistance to the virus strain concern. It is related to the amount of neutralizing antibody (IgA) in the mucus secretions of the respiratory tract as well as to the titre of serum antibodies. When an individual experiences a second or third attack of influenza his humoral antibody response is determined by his previous experience of myxovirus infections usually the infecting strain shares antigens with earlier strains and provokes an anamnestic antibody response to them.

\section{Immunity in Adenovirus}

The immunity that follows adenovirus infection is long lasting and second attack with the same serotype are rare. Neutralizing antibodies to one or more serotypes are present in more than $50 \%$ of children over age 6-11 months. The incidence increases markedly with the age of the individual and between 6-34 years, the majority of the persons have neutralizing antibodies to more than three types Complement antibodies are also commonly present [47].

\section{Immunity in Covid-19}

Covidvirus makes a larger binding interface, as well as more contacts with ACE2. It can make better contact with the N-terminal helix of ACE2 and have higher affinity [48]. It is transmitted through respiratory droplets from coughing and sneezing and enters the nasal system by inhaling and starts replicating. ACE2 is the main receptor for the COVID-19 virus [49]. The spike protein (S protein) present on the surface of COVID-19 is pinched inside the host cell binding to the ACE2 receptor. Here, the enzyme furin is present in the host cell and plays a vital role for the virus to enter, which was absent in covid -19 [50]. . Next, the virus starts to propagate with limited innate immune response and can be detected by nasal swabs. The virus then propagates and reaches the respiratory tract, where it faces a more robust innate immune response. At this stage, the disease is clinically manifest and an innate response cytokine may be predictive of the subsequent clinical course [51].

\section{Antibody Responses in Viral Infections}

Antibodies serve as critical barriers to viral infection. Humoral immunity to a virus is achieved through the dual role of antibodies in communicating the presence of invading pathogens in infected cells to effector cells and interfering with processes essential to the viral lifecycle, chiefly entry into the host cell [52].

\section{Shortened Version of Large Work}

Innate immune mechanisms restrict the early stages of infection and delay spread of virus. is by Interferon, NK cells and Macrophages, As a viral infection proceeds, the adaptive (specific) immune response unfolds. Ab \& complement limit viral spread \& reinfection CD8+ CTLs destroy virus infected cells : CD4+ T cells are a major effector cell population. Viruses have evolved strategies to evade the immune response. Virus latency \& antigenic variation are the most effective mechanisms. Many DNA viruses have strategies to control expression of MHC molecules [53].

\section{Conclusion}

In virus infections, the efficiency of antibodies depends largely on whether the virus passes through the bloodstream in order to reach its target organs. A well known example of a virus that follows such a route is the poliovirus. This crosses the intestinal wall, enters the bloodstream and passes on rare occasions to the spinal cord and brain where it proliferates. Small amounts of antibody in the blood can neutralize the virus before it reaches its target cells in the nervous system. A number of other viruses behave in the same way and pass through the bloodstream on their way to their target organ. Examples are the viruses of of measles, smallpox, mumps, rubella and chickenpox. These viruses have long incubation period. In influenza and common cold, low incubation period is present. In this type of infection a high blood level of antibody will be less effective agaist these viruses in comparison with its effect on bloodborne viruses.

\section{Acknowledgement}

None.

\section{Conflicts of Interest}

No conflicts of interest. 


\section{References}

1. Rehermann B (2009) Hepatitis C virus versus innate and adaptive immune responses: a tale of coevolution and coexistence. J Clin Invest 119: $1745-1754$

2. Favre D, Sharon Lederer, Bittoo Kanwar, Zhong-Min Ma, Sean Proll, et al (2009) Critical loss of the balance between Th17 and T regulatory cell populations in pathogenic SIV infection. PLoS Pathog 5: e1000295.

3. Rowan AG, Jean M Fletcher, Elizabeth J Ryan, Barry Moran, John E Hegarty, et al. (2008) Hepatitis C virus-specific Th17 cells are suppressed by virus-induced TGF- $\beta$. J Immunol 181: 4485-4494.

4. Bermejo-Martin JF, Raul Ortiz de Lejarazu, Tomas Pumarola, Jord Rello, Raquel Almansa, et al. (2009) Th1 and Th17 hypercytokinemia as early host response signature in severe pandemic influenza. Crit Care 13: R201.

5. Culley FJ, Pennycook AM, Tregoning JS, Hussell T, Openshaw PJ (2006) Differential chemokine expression following respiratory virus infection reflects Th1- or Th2-biased immunopathology. J Virol 80: 4521-4527

6. Roubille C (2010) Cytomegalovirus (CMV) and acute myocarditis in an immunocompetent patient. Internal Medicine 49(2): 131-133.

7. Allen J Cann (2012) Principles of Molecular Virology, $5^{\text {th }}$ Ed, Elsevier.

8. Graham Simmons, Mars stone, Michael P Busch (2018) Arbovirus diagnostics;From bad to worse due to expanding Dengue virus vaccination and Zika virus epidemics, Clinical infectious diseases. Infectious disease society of America 66-1181

9. WHO. Guidelines on the quality, safety and efficacy of the dengue tetravalent vaccines (live, attenuated).

10. Sondergaard MJ, Friis MB, Hansen DS, Jorgensen IM (2018) Clinical manifestations in infants and children with Mycoplasma pneumoniae infection. PLoS ONE 13(4): e0195288.

11. Karakuzu Z, Iscimen R, Akalin H, Kelebek Girgin N, Kahveci F, et al (2018) Prognostic Risk Factors in Ventilator-Associated Pneumonia. Med Sci Monit 24: 1321-1328.

12. Evans SS, Repasky EA, Fisher DT (2015) Fever and the thermal regulation of immunity: the immune system feels the heat. Nat Rev Immunol 15(16): 335-349.

13. Dienz O, Rincon M (2009) The effects of IL-6 on CD4 T cell responses Clin Immunol 130: 27-33.

14. Lauder SN, Jones E, Smart K, Bloom A, Williams AS, et al. (2013) Interleukin-6 limits influenza-induced inflammation and protects against fatal lung pathology. Eur J Immunol 43(10): 2613-2625.

15. Yang ML, Wang CT, Yang SJ, Leu CH, Chen SH, et al. (2017). IL-6 ameliorates acute lung injury in influenza virus infection. Sci Rep 7: 43829.

16. Dulsi Fatima Armugam, Immunology, Saras publication, Nagerkoil, Kanyakumari, DT, Tamilnadu, India.

17. CA Dinarello, HA Bernheim, GW Duff, HV Le, TL Nagabhushan, et al (1984) Mechanisms of fever induced by recombinant human interferon J Clin Inves 74(3): 906-913.

18. Stanley MA, Sterling JC (2014) Host responses to infection with human papillomavirus. Curr Probl Dermatol 45: 58-74.

19. Kobayashi A, Weinberg V, Darragh T, Smith-McCune K (2008) Evolving immunosuppressive microenvironment during human cervical carcinogenesis. Mucosal Immunol 1(5): 412-420.

20. Bais AG, Beckmann I, Lindemans J, P C Ewing, C J L M Meijer, et al. (2005) A shift to a peripheral Th2-type cytokine pattern during the carcinogenesis of cervical cancer becomes manifest in CIN/III lesions. J Clin Pathol 58: 1096-1100.

21. Scott ME, Shvetsov YB, Thompson PJ, Brenda Y Hernandez, Xuemei Zhu, et al. (2013) Cervical cytokines and clearance of incident human papillomavirus infection: Hawaii HPV cohort study. Int J Cancer. 133: 1187-1196.
22. Peghini BC, Abdalla DR, Barcelos AC, Teodoro L, Murta EF, et al. (2012) Local cytokine profiles of patients with cervical intraepithelial and invasive neoplasia. Hum Immunol 73(9): 920-926.

23. JN Kline, K Kitagaki (2006) Interferons, in Encyclopedia of Respiratory Medicine.

24. SC Rastogi (2002) Elements of immunology CBS Publishers \&Distributors PVT Ltd.

25. James F Collawn, Etty N, Benveniste (2003) Interferon, in Encyclopedia of the Neurological Sciences.

26. Kidd, P (2003) Th1/Th2 Balance: the hypothesis, its limitations, and implications for health and disease. Alternative Medicine Review 8 (3): 223-246.

27. Parkin J, Cohen B (2001) An overview of the immune system. Lancet. 35 (9270): 1777-1789.

28. Immunoglobulin M, The American Heritage Dictionary of the English Language(Fourth ed.). Houghton Mifflin Company. 2004.

29. Alberts B, Johnson A, Lewis J, Walter P, Raff M, et al. (2002) Chapter 24. Molecular Biology of the Cell (4th ed.). Routledge.

30. Capolunghi F, Rosado MM, Sinibaldi M, Aranburu A, Carsetti R (2013) Why do we need IgM memory B cells?. Immunology Letters. 152(2): 114-120.

31. Williams N, O Connell PR (2008). Chapter 62. Bailey \& Love's Short Practice of Surgery (25th ed.). CRC Press p. 1102

32. Hashimoto G, Wright PF, Karzon DT (1983) Antibody-dependent cellmediated cytotoxicity against influenza virus-infected cells. The Journal of Infectious Diseases 148 (5): 785-794.

33. Pollara Justin, Hart Lydia, Brewer Faraha, Pickerel Joy, Packard Beverly Z, et al. (2011) High-throughput quantitative analysis of HIV-1 and SIVspecific ADCC-mediating antibody responses. Cytometry Part A 79(8): 603-612.

34. Wang W, Erbe AK, HankJA, Morris ZS, Sondel PM (2015) NK Cell-Mediated Antibody-Dependent Cellular Cytotoxicity in Cancer Immunotherapy. Front Immunol 6: 368.

35. Capron M, Kazatchkine, Fischer E, Joseph M, Butterworth AE, et al. (1987) Functional role of the alpha-chain of complement receptor type 3 in human eosinophil-dependent antibody-mediated cytotoxicity against schistosomes. J Immunol 139(6): 2059-2065.

36. Sunil Kumar Mohanty, K Saileela (2014) Text book of immunology, Second Edition, Jaypee Brothers Medical publishers (P) Ltd.

37. Richard D Kalish, Philip W Askenase, Molecular mechanisms of CD8+ T cell-mediated delayed hypersensitivity: Implications for allergies, asthma, and autoimmunity. The journal of Allergy and clinical immunology 103(2): 192-199

38. JH Humphrey, RG White, Immunology for students of Medicine.

39. Abbas AK, Lichtman AH, Pillai S, editors. Cellular and molecular immunology. 8th ed. Philadelphia: Elsevier Saunders;2015

40. David M Knipe, Peter M Howley, et al. Fields virology $6^{\text {th }}$ (ed.) 2.

41. Fairley CK, Hocking JS, Currin LC, et al. (2009) The rapid decline in presentations of genital warts after the implementation of a national quadrivalent human papillomavirus vaccination program for young women. Sex Transm Infect 85(7): 499-502.

42. Laz TH, Rahman M, Berenson AB (2012) An update on human papillomavirus vaccine uptake among 11-17-year-old girls in the US, National health. Interview survey, 2010 vaccine 30(24): 5334-5340.

43. E Jawetz, JL Malnick, EA Edelberg, A Lange review of medical microbiology seventeenth Edition.

44. Joanne M Willey, Linda M, Sherwood Christopher J, Woolverton (2011) Prescott's Microbiology, $8^{\text {th }}$ edn, McGraw-Hill international edn. Printed in Singapore. 
45. Javanavic T, Cupic M, Stanojevic M, Knezevic A, Lazarevic I, et al. (2005) Medcrinski, Facultate University, Beogradu.

46. John Macleod, Davidson's principles and practice of medicine,Fouteenth edition, ELBS. JP Duguid, BP Marmion, RHA Swain, Mackie and McCartney (eds.).

47. J Shang, G Ye, K Shi, Yushun Wan, Chuming Luo, et al. Structural basis of receptor recognition by SARS-CoV-2. Nature, 581 (2020): 221-224.

48. Y Wan, J Shang, R Graham, et al. Receptor recognition by novel coronavirus from Wuhan: an analysis based on decade-long structural studies of SARS J Virol 94 (2020) e00127-220.

49. AC Walls, YJ Park, MA Tortorici, A Wall, AT McGuire, et al. (2020) Structure, function, and antigenicity of the SARS-CoV-2 spike glycoprotein, Cell 181(12): 281-292.
50. NL Tang, PK Chan, CK Wong, Ka-Fai To, Alan Ka-Lun Wu, et al. Early enhanced expression of interferon-inducible protein-10 (CXCL-10) and other chemokines predicts adverse outcome in severe acute respiratory syndrome. Clin Chem 51 (2005): 2333-2340

51. Charles D Murin, Ian A Wilson, Andrew B Ward (2019) Antibody responses to viral infections: a structural perspective across three different enveloped viruses. Nat Microbiol 4(5): 734-747.

52. Yoavanit Srivaro (2016) Immune response to viruses, Allergy \& Immunology, Faculty of Medicine, Chulalongkorn University. 